\title{
Membrane capacitance techniques to monitor granule exocytosis in neutrophils
}

\author{
Karsten Lollike $^{\mathrm{a}, *}$, Manfred Lindau ${ }^{\mathrm{b}}$ \\ ${ }^{a}$ The Granulocyte Research Laboratory, Department of Hematology, Rigshospitalet, L-9322, The Finsen Centre, Rigshospitalet, \\ Blegdamsvej 9, 2100, Copenhagen, Denmark \\ ${ }^{\mathrm{b}}$ School of Applied and Engineering Physics, Cornell University, Ithaca, NY 14853-2501, USA
}

\begin{abstract}
Cell membranes behave like electrical capacitors and changes in cell capacitance therefore reflect changes in the cell area. Monitoring capacitance can thus be used to study dynamic cellular phenomenon involving rapid changes in cell surface, such as exo- and/or endocytosis. In this review focus is on the use of capacitance techniques to study exocytosis in human neutrophils. We compare the whole-cell and the cell-attached capacitance techniques, and we review the complete literature dealing with capacitance measurements in human neutrophils. (C) 1999 Elsevier Science B.V. All rights reserved.
\end{abstract}

Keywords: Cell-attached; Whole-cell; Secretion; Fusion pore; Patch-clamp; Membrane fusion

\section{Introduction}

Exocytosis is a sequence of events leading to fusion of the granule membrane with the plasma membrane. The result of exocytosis is liberation of granule matrix components to the extracellular space and upregulation of membrane bound proteins to the plasma membrane. Both the liberation of matrix molecules and upregulation of membrane bound markers can be used to quantify exocytosis by methods such as ELISA, RIA or FACS (see other chapters in this issue). However, these methods only measure the endpoint of exocytosis and not the dynamic process as such. Furthermore, the sensitivity of these methods is such that usual batch studies

\footnotetext{
* Corresponding author. Tel.: +45-35454888; fax: +4535456727; e-mail: klollike@rh.dk
}

of several (hundred) thousands of cells are employed.

Upon exocytosis the granule membrane is inserted into the plasma membrane and thus the area of the cell increases by the area of the granule. Monitoring changes in plasma membrane area is an efficient way to study exocytosis and endocytosis in single cells. This method, the patch-clamp capacitance technique (Neher and Marty, 1982), and its employment to human neutrophils will be the focus of this review.

\section{Biophysical principles}

Capacitance $(C)$ has the unit of farad $(F)$ and it is a measure of the amount of charge that can be stored in a capacitor at a given voltage. A simple capacitor consists of two parallel conducting plates separated by an insulator. The amount of charge that can be stored in such a capacitor is directly proportional to 
the area of its surfaces $(A)$, the dielectric constant $(\kappa)$, the permittivity of free space $\left(\varepsilon_{0}\right)$, and the distance between the plates $(d)$.

$C=\left(\varepsilon_{0} \kappa A\right) / d$

In the case of biological membranes the two plates are formed by the electrically conducting cytosol and extracellular fluid, separated by an insulator, the phospholipid bilayer. Membranes thus behave electrically as capacitors. Furthermore, since all biological membranes have a similar bilayer composition, Eq. (1) can be simplified to

$C=C_{\mathrm{S}} A$

The capacitance is thus directly proportional to membrane area, depending otherwise only on a constant, the specific capacitance $\left(C_{\mathrm{S}}\right)$, having a unit of $\mathrm{F} / \mathrm{m}^{2}$, or in dimensions appropriate for mammalian cells, $\mathrm{fF} / \mu \mathrm{m}^{2}$.

By measuring the cell's plasma membrane capacitance and correlating this with estimates of plasma membrane area, the specific capacitance has been found to be about $10 \mathrm{fF} / \mu \mathrm{m}^{2}$ (Cole, 1968). Close to recent estimates from patch-clamp capacitance measurements giving $8 \mathrm{fF} / \mu \mathrm{m}^{2}$ or $5 \mathrm{fF} / \mu \mathrm{m}^{2}$ in nerve endings and chromaffin cells, respectively (Rosenboom and Lindau, 1994; Solsona et al., 1998). For neutrophils, we have used a specific capacitance of $8 \mathrm{fF} / \mu \mathrm{m}^{2}$ and found values for granule diameters calculated from capacitance changes to be in agreement with values measured by electron microscopy (Nüße and Lindau, 1988; Lollike et al., 1995).

\section{Techniques to monitor capacitance}

To measure capacitance a voltage signal is imposed onto a cell and the resulting currents charging or discharging the membrane are analyzed. In order to obtain precise measurements the membrane must be charged uniformly over the cell. Using the whole-cell patch-clamp configuration this is best achieved in small round cells without large extensions. The capacitance noise tends to be smaller for small cells due to the smaller plasma membrane area (Lindau and Neher, 1988; Gillis, 1995). Human neutrophils are small round cells, and thus from a theo- retical point of view they are ideal for patch-clamp capacitance measurements.

\section{Whole-cell capacitance measurements}

In the whole-cell configuration potential activators or inhibitors of exocytosis may be introduced into the cell via the patch pipette. The diffusion time constant is proportional to the value of access resistance $\left(R_{\mathrm{A}}\right)$ (Pusch and Neher, 1988). In neutrophils entry of the calcium indicator dye fura- 2 was measured giving diffusion time constants of about 3 $\mathrm{s} / \mathrm{M} \Omega R_{\mathrm{A}}$ (Nüße and Lindau, 1993).

Fig. 1 shows a schematic view of the patch-clamp whole-cell configuration together with a minimal equivalent circuit. The various methods which may be used to measure changes in plasma membrane capacitance by the whole-cell patch-clamp configuration have been reviewed in detail elsewhere (Lindau and Neher, 1988; Lindau, 1991; Gillis, 1995). In neutrophils capacitance measurements have been made using two different methods. Small voltage pulses, typically with a duration of 1-4 ms and -20 $\mathrm{mV}$ amplitude were used and the current response fitted by a single exponential decay (Nüße and Lindau, 1988, 1990, 1993; Nüße et al., 1998). When a voltage pulse of amplitude $V_{0}$ is given as the command voltage, then the current response is given by

$I(t)=\left(I_{0}-I_{\mathrm{SS}}\right) \mathrm{e}^{-t / \tau}+I_{\mathrm{SS}}$

with $I_{0}=V_{0} / R_{\mathrm{A}}, I_{\mathrm{SS}}=V_{0} /\left(R_{\mathrm{A}}+1 / G_{\mathrm{M}}\right)$ and $\tau=$ $C_{\mathrm{M}} /\left(R_{\mathrm{A}}+1 / G_{\mathrm{M}}\right)$. The three elements of the equiv-

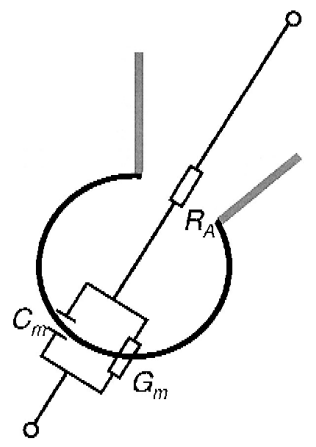

Fig. 1. Schematic drawing of the whole-cell configuration and the equivalent circuit. Think black line indicates cell membrane, gray lines indicates patch pipette. $R_{\mathrm{A}}, G_{\mathrm{M}}, C_{\mathrm{M}}$ are access resistance, membrane conductance, and membrane capacitance, respectively. 
alent circuit $C_{\mathrm{M}}, G_{\mathrm{M}}$ and $R_{\mathrm{A}}$ (membrane capacitance, membrane conductance, and access resistance, respectively) (Fig. 1) can thus be determined from the fit parameters $I_{0}, I_{\mathrm{SS}}$ and $\tau$ (Lindau and Neher, 1988; Lindau, 1991). This method has the advantage that changes in membrane conductance and access resistance can be clearly separated and that even comparatively large conductance changes and shifts of the reversal potential do not interfere with the determination of capacitance changes. The disadvantage of this method is that the total signal power is rather low resulting in relatively high capacitance noise. However, the large capacitance changes occurring during degranulation can still be very well resolved (Fig. 2).

Improved resolution may be obtained if a continuous sine wave is used to charge and discharge the membrane and the resulting sine wave current is analyzed using a lock-in amplifier (Neher and Marty, 1982; Lindau, 1991; Lindau and Neher, 1988; Gillis, 1995). For whole-cell measurements a sine wave frequency of $800 \mathrm{~Hz}$ is typically used. For a detailed discussion of whole-cell capacitance measurements using the lock-in amplifier or phase-sensitive detection technique see (Lindau, 1991; Lindau and Neher, 1988; Gillis, 1995). In principle the method is based on the fact that ohmic currents flowing through the membrane conductance $G_{\mathrm{M}}$ are in phase with the membrane voltage, whereas capacitative currents charging and discharging the membrane capacitance $C_{\mathrm{M}}$ are phase shifted, with respect to the membrane voltage, by $90^{\circ}$. A sine wave command voltage with amplitude $V_{0}$ and frequency $f$ gives rise to a current

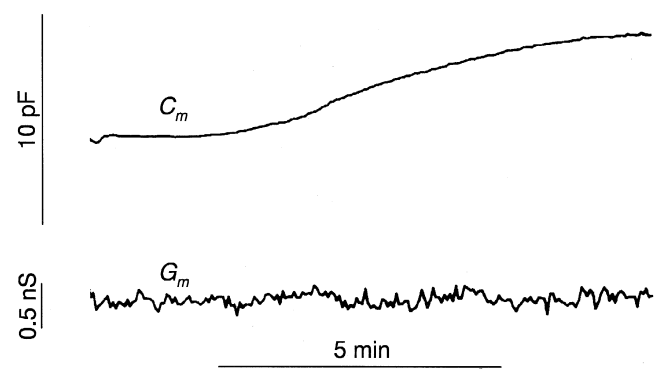

Fig. 2. Trace from whole-cell recording. Whole-cell recording of neutrophil degranulation stimulated by including GPT $\gamma \mathrm{S}$ in the pipette solution (from Nüße and Lindau, 1988). The large capacitance increase (upper trace) occurs while the membrane conductance remains constant (lower trace). with the same frequency which has some amplitude $I_{0}$ proportional to $V_{0}$ and which is phase shifted with respect to the command voltage. The relation between current and voltage can be conveniently expressed using the complex admittance $Y(\omega)$ :

$I=V Y(\omega)$

where $I, V$, and the admittance $Y(\omega)$ are now complex quantities. Choosing the phase of the command voltage as the reference phase is equivalent to defining the command voltage such that its imaginary part is zero:

$V=V_{0} \cos (\omega t)$

The admittance of the equivalent circuit of Fig. 1 is given by (Neher and Marty, 1982)

$$
Y(\omega)=\left(G_{\mathrm{M}}+i \omega C_{\mathrm{M}}\right) /\left(1+R_{\mathrm{A}} G_{\mathrm{M}}+i \omega C_{\mathrm{M}} R_{\mathrm{A}}\right)
$$

The resulting current will thus have not only a real part $(R e)$ but also an imaginary part $(\mathrm{Im})$ corresponding to a component in phase with the voltage and a component shifted by $90^{\circ}$ with respect to the voltage. This measurement provides only two measured quantities, the real and imaginary part of the admittance, and thus does not allow determination of the three equivalent circuit parameters independently. To reconstruct the three parameters the DC current may be used as a third quantity (Lindau and Neher, 1988) or two sine wave frequencies may be used simultaneously (Rohlícek and Rohlicek, 1993; Rohlícek and Schmid, 1994; Donnelly, 1994; Barnett and Misler, 1996).

In neutrophils the piecewise linear technique has been used as originally introduced by Neher and Marty (1982). When small changes of the equivalent circuit parameters are considered, then the admittance change can be linearized:

$\Delta Y \approx \frac{\partial Y}{\partial C_{\mathrm{M}}} \Delta C_{\mathrm{M}}+\frac{\partial Y}{\partial G_{\mathrm{M}}} \Delta G_{\mathrm{M}}+\frac{\partial Y}{\partial R_{\mathrm{A}}} \Delta R_{\mathrm{A}}$

giving

$$
\Delta Y \approx T^{2}(\omega)\left(\Delta G_{\mathrm{M}}+\omega^{2} C_{\mathrm{M}}^{2} \Delta R_{\mathrm{A}}+i \omega \Delta C_{\mathrm{M}}\right)
$$

Besides of the factor $T^{2}(\omega)$ the admittance change has a real part reflecting changes in $G_{\mathrm{M}}$ and $R_{\mathrm{A}}$ and an imaginary part reflecting changes in $C_{\mathrm{M}}$. The term $T^{2}(\omega)$ has a magnitude $\leq 1$ and a phase 
depending on the equivalent circuit parameters. The correct phase setting can be found by setting the capacitance compensation of the patch-clamp amplifier to cancel the cell capacitance. Small changes in the capacitance compensation value can now be made and the phase setting of the lock-in amplifier is set such that these changes appear only in the capacitance trace (Neher and Marty, 1982; Lindau, 1991; Lindau and Neher, 1988; Gillis, 1995). Defined changes of the capacitance compensation (typically 0.1 or $0.2 \mathrm{pF}$ ) may be used to calibrate the capacitance trace. With this method a noise level below 1 fF could be obtained in human neutrophils due to their small resting capacitance of about $3 \mathrm{pF}$, making it possible to observe capacitance steps associated with fusion of individual primary granules (Nüße and Lindau, 1988).

\section{Cell-attached capacitance measurements}

To obtain higher resolution making it possible to resolve exocytosis of smaller granules and secretory vesicles as discrete capacitance steps, the whole-cell configuration cannot be used. The noise is mainly due to the noise generated by the thermal movement of ions in the pipette tip, i.e., the voltage noise due to $R_{\mathrm{A}}$. This voltage noise charges the membrane capacitance, giving rise to a "thermal capacitance noise", proportional to $C_{\mathrm{M}}$ (Lindau and Neher, 1988; Gillis, 1995). The typical plasma membrane capacitance of resting human neutrophils is about $3 \mathrm{pF}$ (Nüße and Lindau, 1988). And the capacitance noise of about 1 fF measured with a $20 \mathrm{mV}$ sine wave is close to the theoretical limit (Gillis, 1995). To further reduce the capacitance noise measurements were made on small membrane patches. This is analogous to conventional single channel recordings. The small noise of patch recordings was essential to provide the high resolution required for detailed characterization of single ion channels carrying small currents (Hamill et al., 1981).

In the pioneering work of Neher and Marty (1982) capacitance steps smaller than $1 \mathrm{fF}$ were observed in cell-attached recordings from chromaffin cells. Fig. 3 shows a schematic diagram of this configuration together with a minimal equivalent circuit. In this

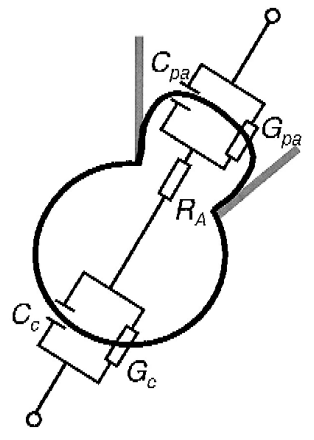

Fig. 3. Schematic drawing of the cell-attached configuration and the equivalent circuit. Thick black line indicates cell membrane, gray lines indicates patch pipette. $R_{\mathrm{A}}, G_{\mathrm{c}}, C_{\mathrm{c}}, G_{\mathrm{pa}}$, and $C_{\mathrm{pa}}$ are access resistance, cell conductance, cell capacitance, patch conductance, and patch capacitance, respectively.

configuration the patch capacitance $C_{\mathrm{pa}}$ and the remaining cell capacitance $C_{\mathrm{C}}$ are connected in series such that, effectively, only the much smaller capacitance $C_{\mathrm{pa}}$ is measured (Lollike et al., 1995). Neher and Marty (1982) obtained very low noise using a $1.6 \mathrm{kHz}$ sine wave with $20 \mathrm{mV}$ amplitude such that capacitance steps down to $0.4 \mathrm{fF}$ could be detected. In more recent studies on human neutrophils we used a $8 \mathrm{kHz}, 50 \mathrm{mV}$ sine wave providing a capacitance noise level of $0.025 \mathrm{fF}$ rms. This allows for resolution of single capacitance steps as small as $0.1 \mathrm{fF}$ (Lollike et al., 1995). A detailed study of the frequency dependence of the capacitance noise has shown that the lowest noise may be obtained in the range of $8-80 \mathrm{kHz}$, however, at frequencies higher than $50 \mathrm{kHz}$ the necessary adjustments become quite tedious (Debus and Lindau, Resolution of patch capacitance recordings and the detectability of fusion pore conductances in small vesicles, submitted).

For neutrophil granules the improved resolution of patch capacitance measurements allows to determine the conductance of the fusion pore, the first connection between the lumen of a vesicle and the extracellular space. When the fusion pore conductance is small, the phase shifted (capacitance) signal is reduced and an in-phase signal appears. Fig. 4 shows an equivalent circuit of a fusing vesicle with capacitance $C_{\mathrm{V}}$ including the fusion pore conductance $G_{\mathrm{P}}$. The admittance change now reflects the change in $G_{\mathrm{P}}$. Assuming that the other parameters 


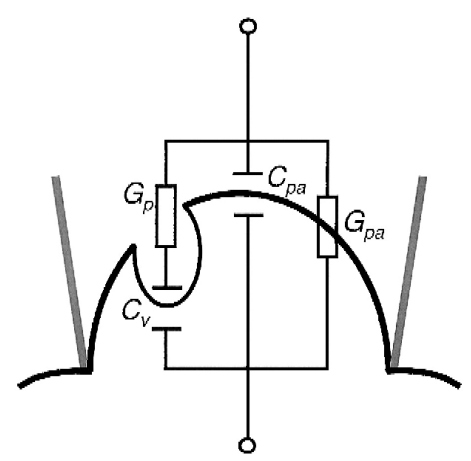

Fig. 4. Equivalent circuit of the patch upon exocytosis and opening of a fusion pore. Thick black line indicates cell membrane, graylines indicates patch pipette. $G_{\mathrm{pa}}, C_{\mathrm{pa}}, G_{\mathrm{p}}$, and $C_{\mathrm{V}}$ are patch conductance, patch capacitance, fusion pore conductance, and vesicle capacitance, respectively.

$\left(C_{\mathrm{pa}}, G_{\mathrm{pa}}, R_{\mathrm{A}}\right.$ and $\left.C_{\mathrm{V}}\right)$ are constant, the admittance change relative to the unfused state $\left(G_{\mathrm{P}}=0\right)$ is given by (Breckenridge and Almers, 1987a; Spruce et al., 1990)

$$
\begin{aligned}
\Delta Y= & T^{2}(\omega)\left(\frac{\left(\omega C_{\mathrm{V}}\right)^{2} / G_{\mathrm{P}}}{1+\left(\omega C_{\mathrm{V}} / G_{\mathrm{P}}\right)^{2}}\right. \\
& \left.+i \frac{\omega C_{\mathrm{V}}}{1+\left(\omega C_{\mathrm{V}} / G_{\mathrm{P}}\right)^{2}}\right)
\end{aligned}
$$

If the phase setting is again set as described for the piecewise linear technique (Eq. (6)), then the lock-in outputs directly provide the two quantities

$$
\begin{aligned}
& R e=\frac{\left(\omega C_{\mathrm{V}}\right)^{2} / G_{\mathrm{P}}}{1+\left(\omega C_{\mathrm{V}} / G_{\mathrm{P}}\right)^{2}} \\
& I m=\frac{\omega C_{\mathrm{V}}}{1+\left(\omega C_{\mathrm{V}} / G_{\mathrm{P}}\right)^{2}}
\end{aligned}
$$

From these measured quantities the equivalent circuit elements $C_{\mathrm{V}}$ and $G_{\mathrm{P}}$ can be calculated (Lindau, 1991; Lollike et al., 1995):

$$
\begin{aligned}
C_{\mathrm{V}} & =\frac{1}{\omega} \frac{R e^{2}+I^{2}}{I m} \\
G_{\mathrm{P}} & =\frac{R e^{2}+I m^{2}}{\operatorname{Re}}
\end{aligned}
$$

It is thus possible to follow the opening and closing of fusion pores, just like the opening and closing of ion channels.

\section{Whole-cell versus cell-attached}

The whole-cell and the cell-attached techniques are in many cases complementary, such that what is an advantage with one technique will be a disadvantage with the other technique, and vice versa (see Table 1). The major setback of the whole-cell technique is the limited resolution. The major setback of the cell-attached technique is that it is impossible to manipulate the cytosol. Furthermore, human neutrophils are very difficult - if not impossible - to microinject (Laffafian and Hallett, 1998). In theory, it should be possible to patch-clamp a human neutrophil with two pipettes. One pipette in the cell-attached configuration that actually makes the electrophysical recording, and one pipette in the whole-cell configuration, not used to measure capacitance, but used to dialyze mediators or inhibitors into the cell. In practice this is probably too heroic. A procedure that seems more feasible is the use of excised patches to gain control of the cytosolic side of the membrane (see perspectives).

\section{Equipment}

Patch-clamp capacitance recordings require highly specialized equipment, which for the most parts are commercially available; for a detailed description of general patch-clamping equipment see Single-Channel Recording (Penner, 1995). For capacitance measurements using voltage pulses standard data acquisition equipment has been used and single exponential fits were performed online using special software. The EPC-9 patch clamp amplifier (HEKA Elektronik, Darmstadt, Germany) has a built in capacitance tracking mode which performs this type of capacitance measurement. For measurements using the lock-in technique any commercially available lock-in amplifier may be used. In addition the operation of a lock-in amplifier has also been performed 
Table 1

Comparison of the whole-cell versus the cell-attached patch-clamp capacitance technique

\begin{tabular}{|c|c|c|}
\hline Configuration & Advantage & Disadvantage \\
\hline \multirow[t]{3}{*}{ Whole-cell } & Total cell response is monitored & $\begin{array}{l}\text { Important cytosolic molecules may be lost by dialysis } \\
\text { into the patch pipette }\end{array}$ \\
\hline & Reagents may be dialyzed into the cell & Exocytosis cannot be separated from endocytosis \\
\hline & $\begin{array}{l}\text { Can easily be combined with intracellular } \mathrm{Ca}^{2+} \text { - } \\
\text { measurements }\end{array}$ & Limited resolution; fusion pores cannot be resolved \\
\hline \multirow[t]{4}{*}{ Cell-attached } & Intact cells are used & Only small part of membrane is monitored \\
\hline & Single exocytic events can be studied & $\begin{array}{l}\text { Only drugs that can penetrate the membrane or drugs } \\
\text { that work from the outside of the plasma membrane, } \\
\text { can be used to stimulate the cells }\end{array}$ \\
\hline & Exocytosis can be separated from endocytosis & \\
\hline & The dynamics of the fusion pore can be studied & \\
\hline
\end{tabular}

by special software running in the data acquisition computer (Joshi and Fernandez, 1988). A widely used lock-in software specifically developed for patch-clamp capacitance measurements that has been developed by Dr. Richard Bookman and his coworkers may be downloaded from the website http://www.instrutech.com/ (Instrutech, Port Washington, NY, USA). For cell-attached measurements, frequencies $\geq 8 \mathrm{kHz}$ should be used this requires a lock-in amplifier. We have most recently used an instrument employing digital signal processing techniques (SR 830 Stanford Research Systems, Sunnyvale, CA).

For pipette fabrication we used hard-glass borosilicate pipettes (Hilgenberg, Malsfeld, Germany) pulled to a diameter of 2.5 to $5 \mathrm{M} \Omega$ and coated with silicone (2-component RTV, GE Silicones, Bergen op Zoom, The Netherlands).

\section{Patch-clamping}

Human neutrophils isolated by standard techniques (Boyum, 1968) can be used directly for capacitance measurements. One advantage of working with human neutrophils is their ability to adhere to glass surfaces such that it is unnecessary to coat the cover slips. In our hands, human neutrophils could still be patched up to $3 \mathrm{~h}$ after isolation when kept on ice. Sealing and rupture of the patch to go whole-cell are performed as in standard patch-clamp (Penner, 1995).
Human neutrophils will appear round as they sediment on the glass cover slip. It is most easy to patch a neutrophil that adheres slightly. Such cells can be located by gently touching the microscope stand; adherent cell will move only little, whereas non-adherent cells will start to rotate on their own axis or float around. Within 5 to $30 \mathrm{~min}$. after adherence the round neutrophils attach more firmly to the cover slip and now assume a "flattened" appearance. Such cells are not easily patched. Human neutrophils can still be very mobile even after patching. Occasionally, neutrophils can flatten from the normal round appearance just to later assume the round appearance again, and this circle of events can repeat itself several times. Such recordings should not be used for analysis because the change in form and adherence to the cover slip may affect the capacitance. Nevertheless, this behavior demonstrates that patched neutrophils are still viable and active.

Fluorescent dyes (e.g., fura-2) can easily be loaded directly into the cells in the whole cell configuration by including the dye in the pipette solution. Capacitance measurements can thus be performed simultaneously with $\mathrm{Ca}^{2+}$ measurements (Nüße and Lindau, 1993; Nüße et al., 1998). When experiments are done in the cell-attached configuration, cells may be pre-loaded with fura- 2 using the ester loading technique. However, it should be kept in mind that the capacitance is measured only in the small patch under the pipette, whereas the calcium measurement is usually performed over the whole cell. Even if the signal is strong enough to use calcium imaging 
technique the local calcium changes near the patch are very hard to determine since the pipette strongly distorts the image in this area.

\section{Obtained results}

Human neutrophils are among the few cells that have been studied by both the whole-cell and the cell-attached capacitance technique. The whole-cell technique have primarily been used to study the effect of second messengers on the exocytic response whereas the cell-attached technique primarily have been used to study the dynamics of the exocytic process as such, and especially the fate of the fusion pore. Nüße and Lindau (Nüße and Lindau, 1988) were the first to apply the whole-cell technique to human neutrophils. They reported an average increase in cell capacitance from $3 \mathrm{pF}$ in resting cells to 8-9 $\mathrm{pF}$ upon stimulation with GTP $\gamma \mathrm{S}$ and low $\mathrm{Ca}^{2+}$ (nM) (applied intracellulary via the patch pipette). They calculated that compared to morphometrical data this capacitance increase matches complete release of primary and secondary granules. In measurements with a lock-in amplifier, they could resolve stepwise increases in capacitance down to 1 $\mathrm{fF}$ corresponding to exocytosis of single primary granules. When stimulating with GTP $\gamma \mathrm{S}$, they found that $\mathrm{Ca}^{2+}$ was not a prerequisite for exocytosis and did not influence the extent of the capacitance increase.

In such experiments GTP $\gamma \mathrm{S}$ stimulates not only degranulation but also a calcium transient as measured using the indicator dye fura-2 (Nüße and Lindau, 1990, 1993). The decay phase of the transient was found to be due to diffusional exchange with the pipette solution. The onset of degranulation was strongly correlated with the time of the calcium increase (Nüße and Lindau, 1993). However, when the calcium change was inhibited by including a calcium buffer at high concentration, the time course of degranulation remained unchanged. Only in experiments where calcium was buffered to values well below resting levels was degranulation somewhat slower (Nüße and Lindau, 1993). It was concluded that degranulation stimulated by intracellular application of GTP $\gamma \mathrm{S}$ is practically independent of changes in intracellular calcium. When cells, patch clamped in the whole-cell configuration, were stimulated with the chemotactic peptide fMLP much shorter calcium transients were observed. These were also coincident with the onset of degranulation and fMLP induced degranulation was found to be more sensitive to calcium buffering (Nüße and Lindau, 1993).

More recently Nüße et al. (1998) have shown, very elegantly, that two phases of exocytosis can be detected by capacitance, when dialyzing the cell with high $\mathrm{Ca}^{2+}$ concentrations. An initial increase in capacitance occurs at $\left[\mathrm{Ca}^{2+}\right]_{\mathrm{i}}$ of 1 to $10 \mu \mathrm{M}$ whereas a second phase calls for a higher concentration of 100 to $300 \mu \mathrm{M} \mathrm{Ca}^{2+}$. Based on morphometric comparisons and the fact that only the second phase of exocytosis abolishes myeloperoxidase staining of individual patched cells, it was concluded that only the high calcium concentrations release primary (azurophilic) granules from human neutrophils. The varying requirement for calcium to initiate exocytosis from different granule fractions seems to correlate with earlier results achieved by other techniques using cell suspensions (Lew et al., 1986; Sengeløv et al., 1993). Nüße et al. conclude that the $\mathrm{Ca}^{2+}$ affinity of exocytosis includes granule-specific elements. It is interesting to note that when using high $\mathrm{Ca}^{2+}$ concentrations $(\mu \mathrm{M})$ the same extent of exocytosis, measured as capacitance increase, can be achieved as when using GTP $\gamma \mathrm{S}$, suggesting that both stimuli are able to stimulate complete degranulation.

By improving the cell-attached capacitance technique (Neher and Marty, 1982) Lollike et al. (1995) were able to obtain a rms capacitance noise level as low as $0.025 \mathrm{fF}$ such that capacitance steps of $0.1 \mathrm{fF}$ could reliably be detected. This corresponds to vesicles with a diameter of $60 \mathrm{~nm}$ and allows for the detection of exocytosis of all granules and secretory vesicles of human neutrophils as single capacitance steps. Following seal formation spontaneous downward capacitance steps were observed indicating endocytosis of small vesicles with diameter of 60-165 nm. A stepwise capacitance increase (Fig. 5) was observed following stimulation with the calcium ionophore Ionomycin (Lollike et al., 1995). Capacitance steps were in the range of $0.1-5 \mathrm{fF}$ consistent with exocytosis of the different granule types of human neutrophils. In vesicles $>180 \mathrm{~nm}$ (step size $>0.8 \mathrm{fF}$ ) the opening of individual fusion pores could be resolved. Fusion pores were found to open 
with an initial conductance of $150 \mathrm{pS}$ (mean, $n=43$ ), although initial fusion pore conductances could be as low as $35 \mathrm{pS}$ (Fig. 6). Subsequently, the fusion pores expanded with variable time course, either rapidly, or sometimes more gradually in distinct phases. This behavior is similar to that of fusion pores observed in giant granules (Breckenridge and Almers, 1987b; Scepek and Lindau, 1993). This was the first time fusion pores could be resolved in granules $<500$ $\mathrm{nm}$ in diameter. The small conductance of the initial fusion pore led the authors to conclude that the early fusion pore has some protein components.

Occasionally fusion pores in neutrophil granules can flicker; i.e., open and close repeatedly (Lollike et al., 1998), as reported for larger granules (Fernandez et al., 1984; Monck et al., 1990), indicating that this late stage in exocytosis is also reversible in small granules. The pore conductance during flicker was generally $<1 \mathrm{nS}$ and the size of the on- and off-steps of flickers were identical. This is in contrast to previous observations in mast cells where fusion pore conductance during flicker is frequently much larger (Spruce et al., 1990) and the size of the off-step exceeds that of the on-step indicating lipid transfer form the plasma membrane to the granule during flicker (Monck et al., 1990). In spite of the markedly improved resolution no significant difference between off- and on-step size was observed in neutrophils. This indicates that the fusion pore does not allow for lipid flux when its conductance is low (Lollike et al., 1998). This agrees well with the finding that in fusion mediated by influenza hemagglutinin lipid flow was only observed if the fusion

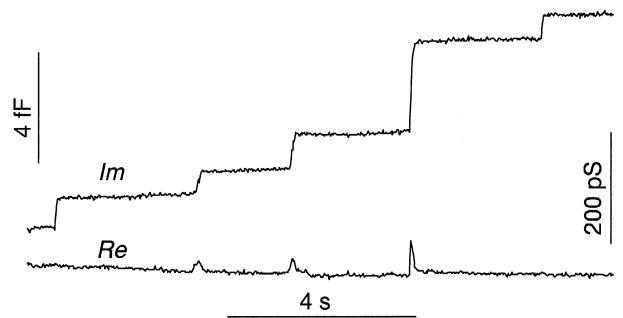

Fig. 5. Trace from cell-attached recording. Cell-attached recording from a human neutrophil stimulated with Ionomycin. The upper trace ( Im) shows stepwise capacitance increases, indicating exocytosis of individual granules. The lower trace $(R e)$ shows transient conductance changes at the time of capacitance steps (for the three middle steps) reflecting the fusion pore opening.

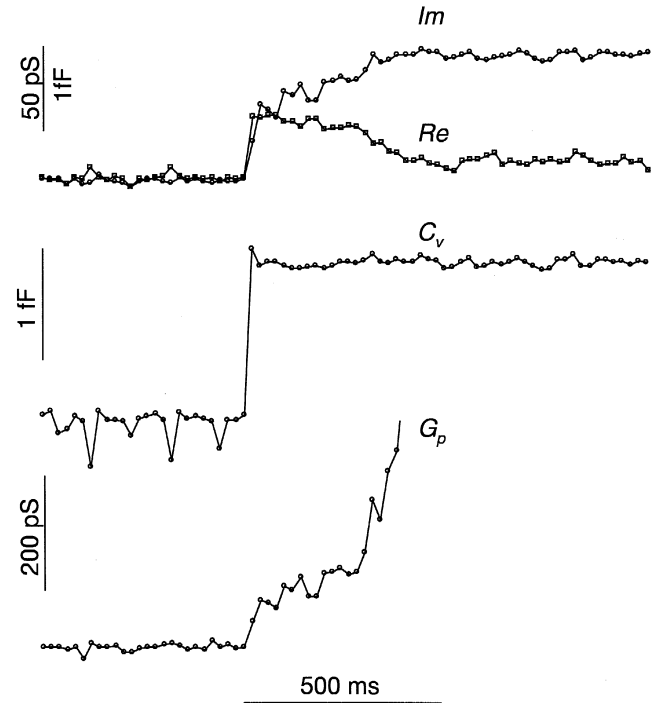

Fig. 6. Resolution of a fusion pore. Analysis of a single fusion event, using Eqs. (10) and (11). Vesicle capacitance $\left(C_{\mathrm{v}}\right)$ and the fusion pore conductance $\left(G_{\mathrm{p}}\right)$ were calculated from changes of the two measured lock-in outputs $\mathrm{Im}$ and $R e$.

pore conductance had exceeded a value of $0.5 \mathrm{nS}$ (Spruce et al., 1990).

Besides regular capacitance flickers Lollike et al. (1998) also observed capacitance increases reflecting single granule fusion events followed by a gradual decline which could not be explained by re-closing of the fusion pore. The explanation for theses "pseudoflickers" is not yet clear. It was proposed that the gradual decrease might reflect rapid endocytosis of several vesicles $<60 \mathrm{~nm}$ (which cannot be clearly resolved as steps). Alternatively, it might reflect attachment of extra membrane, added to the patch in a fusion event, to the pipette wall. Anyway, nonstepwise capacitance changes must be interpreted with caution in the cell-attached configuration (Lollike et al., 1998).

\section{Perspectives}

The whole-cell capacitance technique is one of the few methods to study the dynamics of exocytosis in single cells and provides unique insights into the mechanisms underlying cell signalling. Combination of whole-cell capacitance measurements with fluori- 
metric detection of second messengers allows investigation of these relations in fine detail. The method is now well-established in human neutrophils and may be employed routinely for measurement of exocytosis. It is well suited for studies of signal transduction, and compounds thought to enhance or inhibit certain signalling pathways or the exocytic machinery itself may be evaluated.

The cell-attached patch capacitance technique allows investigation of single exocytotic events for vesicles as small as $60 \mathrm{~nm}$. Thus all the secretory granules and vesicles may be studied with this technique. A basic characterization of the opening of exocytotic fusion pores in neutrophils has been published (Lollike et al., 1995). However, further details of the fusion pores need to be characterized, in particular the dynamics of fusion pore expansion has not been analyzed in great detail yet. Whole-cell fusion pore measurements on horse eosinophils, which have giant granules, revealed that the dynamics of fusion pore expansion is modulated by calcium and protein kinase $\mathrm{C}$ via distinct mechanisms (Hartmann and Lindau, 1995; Scepek et al., 1998). The fusion pore in granulocytes thus appears to be under control of proteins even in an expanded state. Similar studies on neutrophils using patch capacitance measurements have not been undertaken.

While much knowledge of the fusion pore have been learned by capacitance measurements very limited knowledge have been obtained of the fission pore (Rosenboom and Lindau, 1994; Suss-Toby et al., 1996). This is because endocytic vesicles in general are too small to be resolved by the whole-cell technique. However, endocytosis of individual vesicles can be resolved by the cell-attached technique (Lollike et al., 1995) and recently Lollike et al. (1997) have been able to resolve fission pores of small endocytic vesicles (range 300 to $700 \mathrm{~nm}$ ) in a manner similar to fusion pores. This should bring important data on the dynamics of fission pores.

In the cell-attached configuration, the calcium concentration or other modulators of fusion or fission cannot be controlled directly at the cytoplasmic side of the membrane. A next step should thus be to investigate exocytotic fusion events in excised patches. Encouraging results in this direction were the recent observations of exocytotic events and resolution of fusion pores in excised patches from chromaffin cells (Dernick et al., 1999) and human neutrophils (Lollike et al., 1999). This holds promise for an in vitro fusion assay with ms time resolution, such an assay might be very important in bringing the final pieces of the fusion pore puzzle together.

\section{Acknowledgements}

This work has been supported by grants to KL from the Danish Medical Research Council, the Novo Nordisk Fund, Mrs. Astrid Thaysens Fund, and Danish Hospital Foundation for Medical Research; Region of Copenhagen, The Faroe Islands, and Greenland.

\section{References}

Barnett, D.W., Misler, S., 1996. An optimized approach to membrane capacitance estimation using dual frequency excitation. Biophys. J. 72, 1641.

Boyum, A., 1968. Isolation of mononuclear cells and granulocytes from human blood. Scand. J. Clin. Lab. Invest. 21, 77, suppl.

Breckenridge, L.J., Almers, W., 1987a. Currents through the fusion pore that forms during exocytosis of a secretory vesicle. Nature 328, 814.

Breckenridge, L.J., Almers, W., 1987b. Final steps in exocytosis observed in a cell with giant secretory granules. Proc. Natl. Acad. Sci. USA 84, 1945.

Cole, S., 1968. Membranes, Ions and Impulses. University of California Press, Berkeley.

Dernick, G., de Toledo, G.A., Lindau, M., 1999. Capacitance steps indicating endocytosis, exocytosis and fusion pore conductance in cell-free excised patches from chromaffin cells. Biophys. J. 76, Abstract.

Donnelly, D.F., 1994. A novel method for rapid measurement of membrane resistance, capacitance, and access resistance. Biophys. J. 66, 873.

Fernandez, J.M., Neher, E., Gomperts, B.D., 1984. Capacitance measurements reveal stepwise fusion events in degranulating mast cells. Nature 312, 453

Gillis, K.D., 1995. Techniques for membrane capacitance measurements. In: Sakmann, B., Neher, E. (Eds.), Single-Channel Recording, Vol. 2. Plenum, New York, p. 155.

Hamill, O.P., Marty, A., Neher, E., Sakmann, B., Sigworth, F.J., 1981. Improved patch-clamp techniques for high-resolution current recording from cells and cell-free membrane patches. Pflügers Arch 391, 85.

Hartmann, J., Lindau, M., 1995. A novel $\mathrm{Ca}^{2+}$-dependent step in exocytosis subsequent to vesicle fusion. FEBS Lett. 363, 217.

Joshi, C., Fernandez, J.M., 1988. Capacitance measurements. An analysis of the phase detector technique used to study exocytosis and endocytosis. Biophys. J. 53, 885. 
Laffafian, I., Hallett, M.B., 1998. Lipid-assisted microinjection: Introducing material into the cytosol and membranes of small cells. Biophys. J. 75, 2558.

Lew, D.P., Monod, A., Waldvogel, F.A., Dewald, B., Baggiolini, M., Pozzan, T., 1986. Quantitative analysis of the cytosolic free calcium dependency of exocytosis from three subcellular compartments in intact human neutrophils. J. Cell Biol. 102, 2197.

Lindau, M., 1991. Time-resolved capacitance measurements: monitoring exocytosis in single cells. Rev. Biophys. 24, 75.

Lindau, M., Neher, E., 1988. Patch-clamp techniques for time-resolved capacitance measurements in single cells. Pflügers Arch 411, 137.

Lollike, K., Borregaard, N., Lindau, M., 1995. The exocytic fusion pore of small granules has a conductance similar to an ion channel. J. Cell Biol. 129, 99.

Lollike, K., Borregaard, N., Lindau, M., 1997. The fission pore dynamics of small endocytic vesicles resolved by cell-attached capacitance measurements. Mol. Biol. Cell 8, Suppl., Abstract.

Lollike, K., Borregaard, N., Lindau, M., 1998. Capacitance flickers and pseudoflickers of small granules, measured in the cell-attached configuration. Biophys. J. 75, 53.

Lollike, K., Lindau, M., Borregaard, N., Calafat, J., 1999. Resting human neutrophils do not have docked granules, but morphological and functional docking can be induced by stimulation. Eur. J. Clin. Invest. 29, Suppl. 1, Abstract.

Monck, J.R., de Toledo, G.A., Fernandez, J.M., 1990. Tension in secretory granule membranes causes extensive membrane transfer through the exocytotic fusion pore. Proc. Natl. Acad. Sci. USA 87, 7804.

Neher, E., Marty, A., 1982. Discrete changes of cell membrane capacitance observed under conditions of enhanced secretion in bovine adrenal chromaffin cells. Proc. Natl. Acad. Sci. USA 79,6712 .

Nüße, O., Lindau, M., 1988. The dynamics of exocytosis in human neutrophils. J. Cell Biol. 107, 2117.

Nüße, O., Lindau, M., 1990. GTPgammaS-induced calcium transients and exocytosis in human neutrophils. Biosci. Rep. 10, 93.

Nüße, O., Lindau, M., 1993. The calcium signal in human neutrophils and its relation to exocytosis investigated by patch- clamp capacitance and Fura-2 measurements. Cell Calcium 14, 255.

Nüße, O., Serrander, L., Lew, D.P., Krause, K.-H., 1998. Ca ${ }^{2+}$-induced exocytosis in individual human neutrophils: high- and low-affinity granule populations and submaximal responses. EMBO J. 17, 1279.

Penner, R., 1995. A practical guide to patch clamping. In: Sakmann, B., Neher, E. (Eds.), Single-Channel Recording. Vol. 2. Plenum, New York, p. 3.

Pusch, M., Neher, E., 1988. Rates of diffusional exchange between small cells and a measuring patch pipette. Pflügers Arch 411, 204.

Rohlícek, V., Rohlicek, J., 1993. Measurements of membrane capacitance and resistance of single cells with two frequencies. Physiol. Res. 42, 423.

Rohlícek, V., Schmid, A., 1994. Dual-frequency method for synchronous measurements of cell capacitance, membrane conductance and access-resistance on single cells. Pflügers Arch 428, 30 .

Rosenboom, H., Lindau, M., 1994. Exo-endocytosis and closing of the fission pore during endocytosis in single pituitary nerve terminals internally perfused with high calcium concentrations. Proc. Natl. Acad. Sci. USA 91, 5267.

Scepek, S., Lindau, M., 1993. Focal exocytosis by eosinophils compound exocytosis and cumulative fusion. EMBO J. 12, 1811.

Scepek, S., Coorssen, J.R., Lindau, M., 1998. Fusion pore expansion in horse eosionphils is modulated by $\mathrm{Ca}^{2+}$ and protein kinase $\mathrm{C}$ via distinct mechanisms. EMBO J. 17, 4340.

Sengeløv, H., Kjeldsen, L., Borregaard, N., 1993. Control of exocytosis in early neutrophil activation. J. Immunol. 150, 1535 .

Solsona, C., Innocenti, B., Fernandez, J.M., 1998. Regulation of exocytic fusion by cell inflation. Biophys. J. 74, 1061.

Spruce, A.E., Breckenridge, L.J., Lee, A.K., Almers, W., 1990. Properties of the fusion pore that forms during exocytosis of a mast cell secretory vesicle. Neuron 4, 643.

Suss-Toby, E., Zimmerberg, J., Ward, G.E., 1996. Toxoplasma invasion: The parasitophorous vacuole is formed from host cell plasma membrane and pinches off via a fission pore. Proc. Natl. Acad. Sci. USA 93, 8413. 